\title{
Video Article \\ Cooling or Warming the Esophagus to Reduce Esophageal Injury During Left Atrial Ablation in the Treatment of Atrial Fibrillation
} \author{
Erik Kulstad ${ }^{11}$ \\ ${ }^{1}$ St. David's South Austin Medical Center \\ ${ }^{2}$ St George's University Hospitals NHS Foundation Trust, St. George's, University of London \\ ${ }^{3}$ Hospital of the University of Pennsylvania, Perelman Center for Advanced Medicine \\ ${ }^{4}$ University of Pennsylvania Perelman School of Medicine \\ ${ }^{5}$ Hospital de la Santa Creu I Sant Pau, Universitat Autònoma de Barcelona, CIBERCV \\ ${ }^{6}$ PeaceHealth Medical Group, St. Joseph Medical Center \\ ${ }^{7}$ St. Vincent Hospital \\ ${ }^{8}$ University of lowa \\ ${ }^{9}$ University Hospital Erlangen \\ ${ }^{10}$ NorthShore University Health System \\ ${ }^{11}$ Department of Emergency Medicine, University of Texas, Southwestern Medical Center
}

Jason Zagrodzky ${ }^{1}$, Mark M. Gallagher ${ }^{2}$, Lisa W. M. Leung ${ }^{2}$, Tiffany Sharkoski ${ }^{3}$, Pasquale Santangeli ${ }^{4}$, Cory Tschabrunn ${ }^{4}$, Jose M. Guerra ${ }^{5}$, Bieito Campos ${ }^{5}$, John MacGregor ${ }^{6}$, Jamal Hayat ${ }^{2}$, Brad Clark ${ }^{7}$, Alex Mazur ${ }^{8}$, Marcel Feher ${ }^{9}$, Martin Arnold ${ }^{9}$, Mark Metzl ${ }^{10}$, Jose Nazari ${ }^{10}$,

Correspondence to: Jason Zagrodzky at jzagrodzkyep@gmail.com, Erik Kulstad at erik.kulstad@utsouthwestern.edu

URL: https://www.jove.com/video/60733

DOI: doi:10.3791/60733

Keywords: Medicine, Issue 157, atrial fibrillation, radiofrequency ablation, cryoablation, esophageal injury, esophageal cooling, esophageal warming, atrioesophageal fistula

Date Published: 3/15/2020

Citation: Zagrodzky, J., Gallagher, M.M., Leung, L.W.M., Sharkoski, T., Santangeli, P., Tschabrunn, C., Guerra, J.M., Campos, B., MacGregor, J., Hayat, J., Clark, B., Mazur, A., Feher, M., Arnold, M., Metzl, M., Nazari, J., Kulstad, E. Cooling or Warming the Esophagus to Reduce Esophageal Injury During Left Atrial Ablation in the Treatment of Atrial Fibrillation. J. Vis. Exp. (157), e60733, doi:10.3791/60733 (2020).

\section{Abstract}

Ablation of the left atrium using either radiofrequency (RF) or cryothermal energy is an effective treatment for atrial fibrillation (AF) and is the most frequent type of cardiac ablation procedure performed. Although generally safe, collateral injury to surrounding structures, particularly the esophagus, remains a concern. Cooling or warming the esophagus to counteract the heat from RF ablation, or the cold from cryoablation, is a method that is used to reduce thermal esophageal injury, and there are increasing data to support this approach. This protocol describes the use of a commercially available esophageal temperature management device to cool or warm the esophagus to reduce esophageal injury during left atrial ablation. The temperature management device is powered by standard water-blanket heat exchangers, and is shaped like a standard orogastric tube placed for gastric suctioning and decompression. Water circulates through the device in a closed-loop circuit, transferring heat across the silicone walls of the device, through the esophageal wall. Placement of the device is analogous to the placement of a typical orogastric tube, and temperature is adjusted via the external heat-exchanger console.

\section{Video Link}

The video component of this article can be found at https://www.jove.com/video/60733/

\section{Introduction}

Left atrial ablation to perform pulmonary vein isolation $(P V I)$ is increasingly utilized for the treatment of atrial fibrillation ${ }^{1}$. The attainment of PVI can be achieved with radiofrequency (RF) energy to burn atrial tissue or with direct application of cryothermal energy; however, collateral damage to surrounding structures remains a risk with either method, with esophageal injury being one of the most serious ${ }^{2,3,4}$. The most extreme esophageal injury, atrioesophageal fistula (AEF), remains challenging to prevent and diagnose, and carries a very high mortality ${ }^{5,6}$

A number of techniques have been utilized to reduce the risk of $A E F$, including reducing power applied to vulnerable regions, monitoring luminal esophageal temperature (LET), deviating the esophagus during ablation, and cooling or warming the esophagus ${ }^{7}$. Directly countering the thermal energy delivered to the esophagus, primarily by cooling against the RF heating, has been used in a variety of formats ${ }^{8,9,10,11,12,13,14,15,16}$ An advantage to cooling during RF ablation or warming during cryoablation is that a preventive approach to injury is taken, in contrast to temperature monitoring, which involves a reactive approach (stopping ablation when temperature rises). The reactive approach, although often used, may be of limited efficacy ${ }^{17}$, with a recent review noting that currently available discrete sensor probes, whether single or multiple, do not appear to significantly reduce injury rates ${ }^{7}$. Cooling or warming also avoids the need for procedural pauses and device manipulation required with esophageal deviation techniques, which have been reported to cause esophageal trauma and involve difficulties in use ${ }^{18,19}$. $\mathrm{A}$ 
recent meta-analysis of esophageal cooling for the purpose of protecting the esophagus during RF ablation found a $61 \%$ reduction in high-grade lesion formation in a total of 494 patients ${ }^{20}$. A recent randomized-controlled trial found a statistically significant $83 \%$ reduction in endoscopically identified lesions when using a dedicated cooling device compared to standard LET monitoring ${ }^{21}$.

The goal of this protocol is to demonstrate the use of esophageal cooling or warming during left atrial radiofrequency or cryo-ablation using an esophageal temperature management device (Figure 1).

\section{Protocol}

This protocol follows the guidelines of local institution's human research ethics committee where applicable.

\section{Assessment Prior to Placement}

NOTE: Under current U.S. labeling, there are no formal contraindications listed. In the case of esophageal pathology, such as deformity, trauma, or recent ingestion of caustics or acidic material, caution is advised.

1. Ensure that necessary equipment, such as the heat exchanger, the esophageal temperature management device, and water-based lubrication, is available.

2. Attach the esophageal temperature management device to the heat exchanger via the device connectors, and power on the unit, placing it in manual mode. Ensure that the water is flowing through the esophageal temperature management device and confirm the absence of leaks.

\section{Placement}

1. Determine the appropriate insertion depth for the esophageal temperature management device in similar manner to standard orogastric tube. Measure from the patient's lips to the earlobe and from the earlobe to xiphoid process and note this depth on the device (Figure 2).

2. Use water-soluble lubricant to lubricate the esophageal temperature management device generously, at least $15 \mathrm{~cm}$, and up to $25 \mathrm{~cm}$ of the distal end (Figure 3).

NOTE: Patients are typically under general inhalational anesthesia (for example, using sevoflurane), but may also be under intravenous anesthesia (for example, using propofol), or in some cases under conscious sedation (for example, using meperidine or midazolam).

3. If possible, extend the patient's head to further facilitate insertion of the esophageal temperature management device using gentle pressure applied posteriorly and downwards, past the oropharynx and into the esophagus. Lifting the mandible anteriorly may assist passage of the device, as might a reduction of pressure in the ETT cuff if overinflated. Apply light pressure on the device as needed to reach the desired depth of placement. (Figure 4).

4. Determine placement location by fluoroscopy to check if the tip of the device is below the diaphragm (Figure 5).

5. Secure the water hoses and device to avoid accidental dislodgement; a common method is to place the connecting hose under the patient's left foam armrest.

6. If stomach decompression is desired, connect the central lumen to low-intermittent suction using standard suction tubing

\section{Temperature Modulation - RF Ablation}

1. Ensure that the heat exchanger is set to manual mode and the appropriate water temperature is set. For example, on one typical heat exchanger, press the Temp Control button, then use the up/down arrows to select the target water temperature. Once the digital display shows the target temperature desired, initiate water flow by pressing the Manual Control button. A typical target is $4{ }^{\circ} \mathrm{C}$ water temperature when performing radiofrequency ablation at the posterior left atrial wall.

2. In order to anticipate the time needed for the heat exchanger to reduce temperature, use a water temperature setpoint of roughly $14{ }^{\circ} \mathrm{C}$ for initial insertion in RF cases while awaiting transseptal puncture. After transseptal puncture, and approximately 15-20 min before application of RF energy to the posterior atrial wall, change the water temperature setpoint to $4{ }^{\circ} \mathrm{C}$ (in manual mode).

NOTE: For additional anti-inflammatory effects of cooling which may reduce gastroparesis or chest pain post-procedure, operators may maintain the water temperature setpoint at $4{ }^{\circ} \mathrm{C}$ for $20 \mathrm{~min}$ after the completion of posterior wall ablation, at which point the machine can be turned off.

\section{Temperature Modulation - Cryoablation}

1. For cryoablation, use a water temperature setpoint of $42^{\circ} \mathrm{C}$ (typical).

2. Set this water temperature shortly after placement (placing while cold is generally easier due to increased device stiffness), and continue throughout the case, providing additional patient warming to counter the systemic cooling effect of the cryoablation.

\section{Patient Temperature Monitoring}

NOTE: Because the temperature in the esophagus is modulated by the presence of an esophageal heat transfer device, a different location is necessary for patient temperature measurement. Options for patient temperature measurement include nasopharyngeal thermometer (ensure that the depth is less than $10 \mathrm{~cm}$ ), Foley temperature sensor, rectal temperature sensor, tympanic membrane thermometer, or forehead thermometer (including zero-flux thermometry). 
1. To maintain patient temperature when using esophageal cooling, use supplemental warming modalities, such as warming blankets or head covers if needed. During esophageal warming when performing cryoablation, the patient temperature will usually stay in a normothermic range.

\section{Troubleshooting}

1. Ensure that no blockage of water flow occurs, and that the water paddle wheel, if present, is continuously spinning, or the low-flow alarm is not activated.

2. Blockage of water flow in the system will cause the paddle wheel to stop spinning and an occlusion alert on the external heat exchanger Stop treatment and determine the location and cause of obstruction. If necessary, remove and replace the esophageal temperature management device.

3. Confirm water flow at correct temperature by checking setpoint and touching device to ensure adequate pressure (device will be firm) and appropriate temperature.

\section{Removal of Device}

1. Press the appropriate button to pause water flow; this may be labelled "Monitor" or "Temp Set", but may vary by model.

2. If present, close clamps on the hose set and/or device tubing, and withdraw the device from the patient by gently pulling anteriorly in a similar manner to standard orogastric tube removal.

3. Power down the heat exchange unit via the power switch prior to unplugging from wall power.

4. Disconnect the water hose connectors from the device and dispose as per institutional policy (typically via contaminated waste container).

\section{Representative Results}

A large number of patients have been studied using esophageal cooling via direct instillation of cold liquid into the esophagus during RF ablation (for example, by injecting a $20 \mathrm{~mL}$ bolus of ice-cold saline via orogastric tube into the upper esophagus when the LET increased by $0.5^{\circ} \mathrm{C}$ above baseline). The findings of a meta-analysis of existing studies using this technique is summarized in Figure $6^{20}$.

Data from a randomized-controlled clinical trial evaluating a dedicated cooling device were recently presented, and are summarized in Table $\mathbf{1}^{21}$. Ablation parameters for the control and treatment arms, respectively, were as follows: RF duration, 14.1 versus 14.5 min; average force, 19.1 versus $17.8 \mathrm{~g}$, maximum RF power, 33.9 versus $34.1 \mathrm{~W}$, and average ablation index, 394 versus 384, with all differences non-significant. All patients had PVI with additional lesion sets when required. At the time of presentation, no difference in recurrence rate of atrial fibrillation at 6 months was found between the two groups (4/27 in control group, 3/17 in treatment group).

Example RF ablation result:

A 59 year-old female with a past medical history of hyperlipidemia, diabetes, and recurrent paroxysmal atrial fibrillation presented for an RF ablation procedure. An esophageal heat transfer device circulating $14{ }^{\circ} \mathrm{C}$ water was placed in the esophagus, with the setpoint reduced to $4{ }^{\circ} \mathrm{C}$ after transseptal puncture, approximately $8 \mathrm{~min}$ before the start of ablation. The ablation was performed using a three-dimensional mapping system and a $3.5 \mathrm{~mm}$ irrigated ablation catheter for segmental pulmonary vein isolation. A setting of $30 \mathrm{~W}$ on the posterior aspect of the pulmonary veins, with up to $40 \mathrm{~W}$ on the anterior was used, with duration of up to $20 \mathrm{~s}$. PVI as well as linear posterior wall isolation (Box lesion) was performed. Patient temperature was measured via nasopharyngeal probe placed less than $10 \mathrm{~cm}$ into the nares, with patient start temperature of $36.4{ }^{\circ} \mathrm{C}$, and end temperature of $36.1{ }^{\circ} \mathrm{C}$. Approximately $20 \mathrm{~min}$ after completion of ablation on the posterior wall, the esophageal heat transfer device setpoint was raised to $40^{\circ} \mathrm{C}$ to provide patient warming while access sheaths were removed and vascular closure was completed. Endoscopy performed the following day as part of a research protocol demonstrated no esophageal lesions.

Example cryoablation result:

A 68 year-old male with past medical history of hypertension and increasing episodes of paroxysmal atrial fibrillation presented for cryoballoon ablation. An esophageal heat transfer device circulating room temperature $\left(22^{\circ} \mathrm{C}\right)$ water was placed in the esophagus. Once placed, the setpoint temperature was raised to $42^{\circ} \mathrm{C}$. Ablations were performed with a cryoballoon system. Initial patient core temperature was measured at 36.3 ${ }^{\circ} \mathrm{C}$ via Foley catheter temperature sensor. Temperatures in the esophagus were measured with a single-sensor temperature probe (routine use of a temperature probe device co-located with the heat transfer device is not recommended, as the optimal benefit is obtained with full contact between heat transfer device and esophageal mucosa, but is described here to show the effect on preventing excessive temperature decreases). Beginning with cryoablation at the left superior pulmonary vein, the initial esophageal temperature measured was $38.6^{\circ} \mathrm{C}$ and reached a nadir of $36.4^{\circ} \mathrm{C}$ during the cryoablation. Nadir balloon temperature was $-51^{\circ} \mathrm{C}$. Block was obtained in under $30 \mathrm{~s}$, with a single 180 second freeze performed. At the left inferior pulmonary vein, the beginning temperature was $38.5^{\circ} \mathrm{C}$ and reached a low of $38.0^{\circ} \mathrm{C}$ after two cycles of treatment (a bonus freeze of $120 \mathrm{~s}$ was performed because of delay in obtaining block on initial freeze until $70 \mathrm{~s}$ in). Nadir balloon temperature was $-48{ }^{\circ} \mathrm{C}$. In the right superior pulmonary vein, initial esophageal temperature was $38.4^{\circ} \mathrm{C}$, remained unchanged through two cycles, and ended at $38.5^{\circ} \mathrm{C}$. Nadir balloon temperature was $-47^{\circ} \mathrm{C}$. Finally, in the right inferior pulmonary vein, initial esophageal temperature was $38.9^{\circ} \mathrm{C}$ and reached a nadir of $38.8^{\circ} \mathrm{C}$ throughout two cycles of treatment. Nadir balloon temperature was $-39^{\circ} \mathrm{C}$. Patient temperature at the end of the procedure was $36.0^{\circ} \mathrm{C}$, and all cryoballoon treatments maintained esophageal temperature well above common stopping thresholds $\left(15^{\circ} \mathrm{C}\right.$ to $\left.25^{\circ} \mathrm{C}\right)$. 


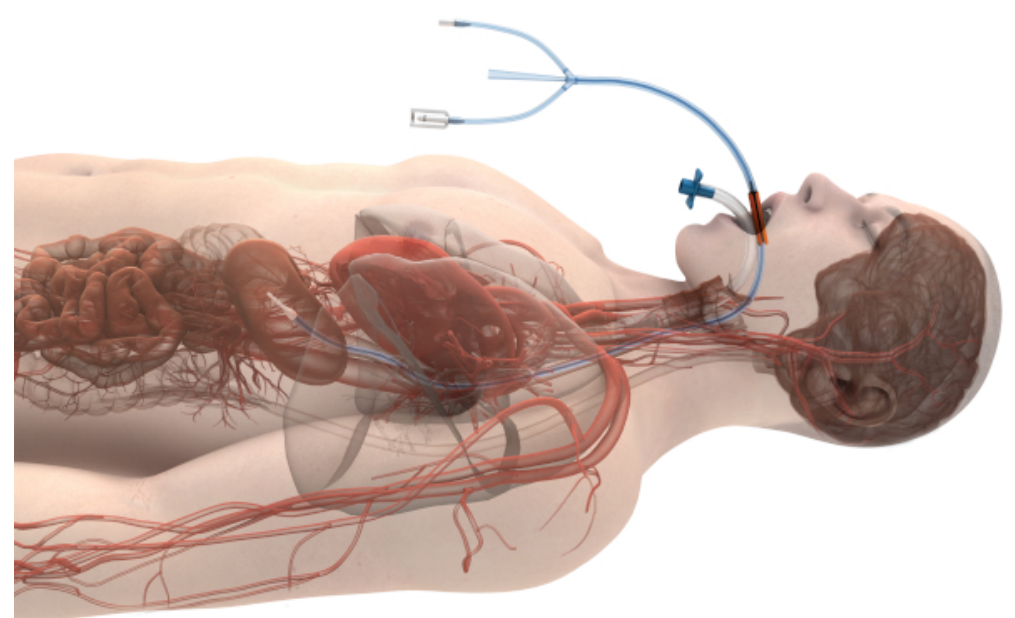

Figure 1: Image of esophageal temperature management device in-situ (with permission from Attune Medical). Please click here to view a larger version of this figure.

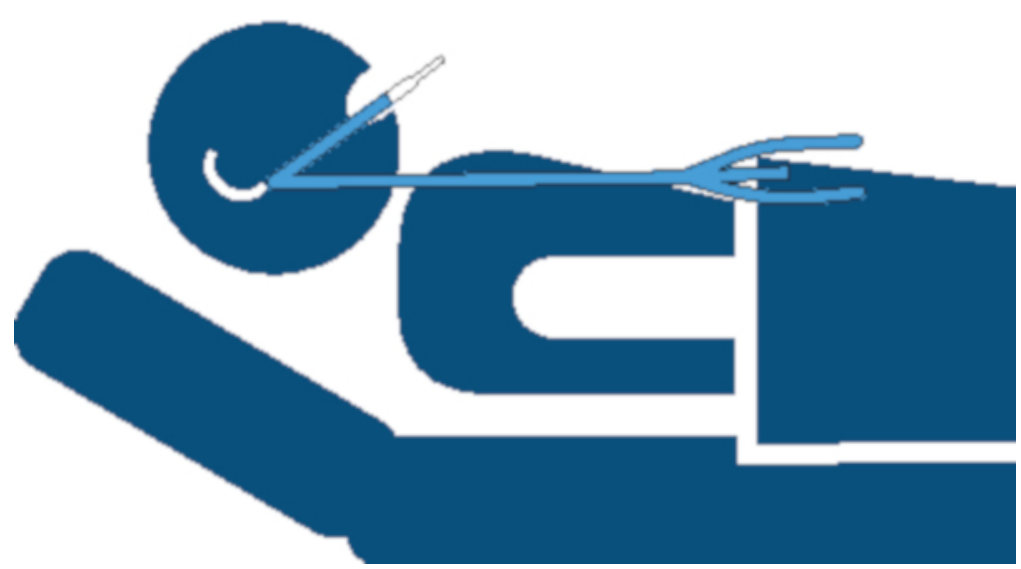

Figure 2: Measurement of the appropriate insertion depth for the esophageal temperature management device. This is performed by extending the device from the patient's lips to the earlobe and then from the earlobe to the tip of the xiphoid process, and then marking the insertion depth on the device. Please click here to view a larger version of this figure.

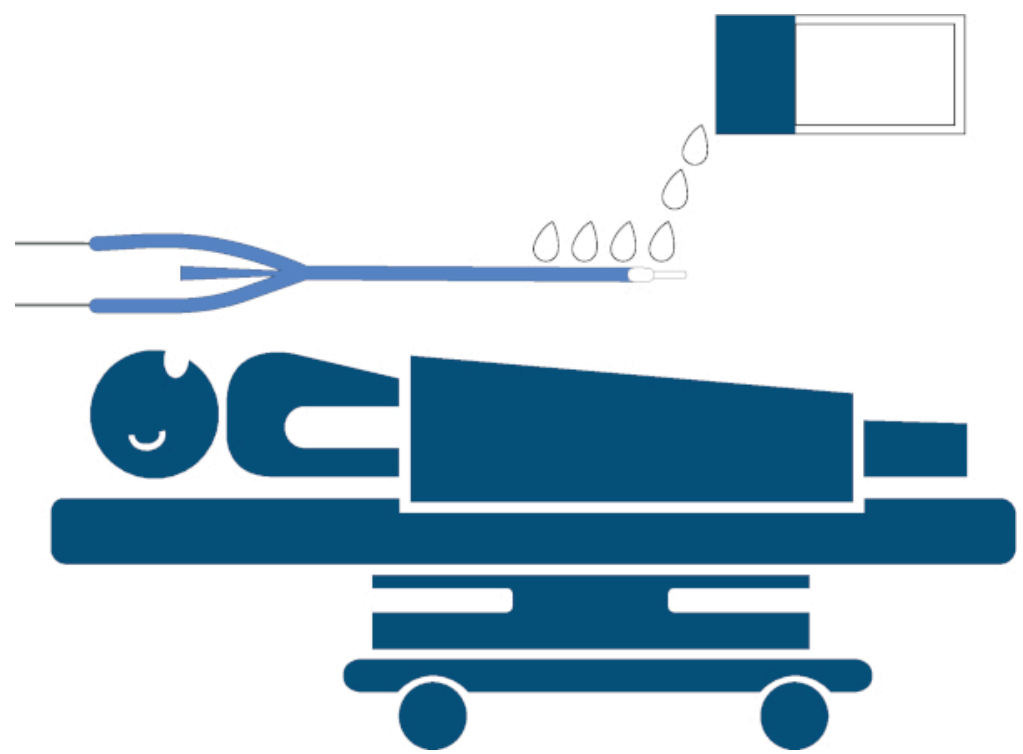

Figure 3: Lubrication of the device. Lubrication of the esophageal temperature management device, generously applying approximately lubricant to $25 \mathrm{~cm}$ of the distal end with water-soluble lubricant. Please click here to view a larger version of this figure. 


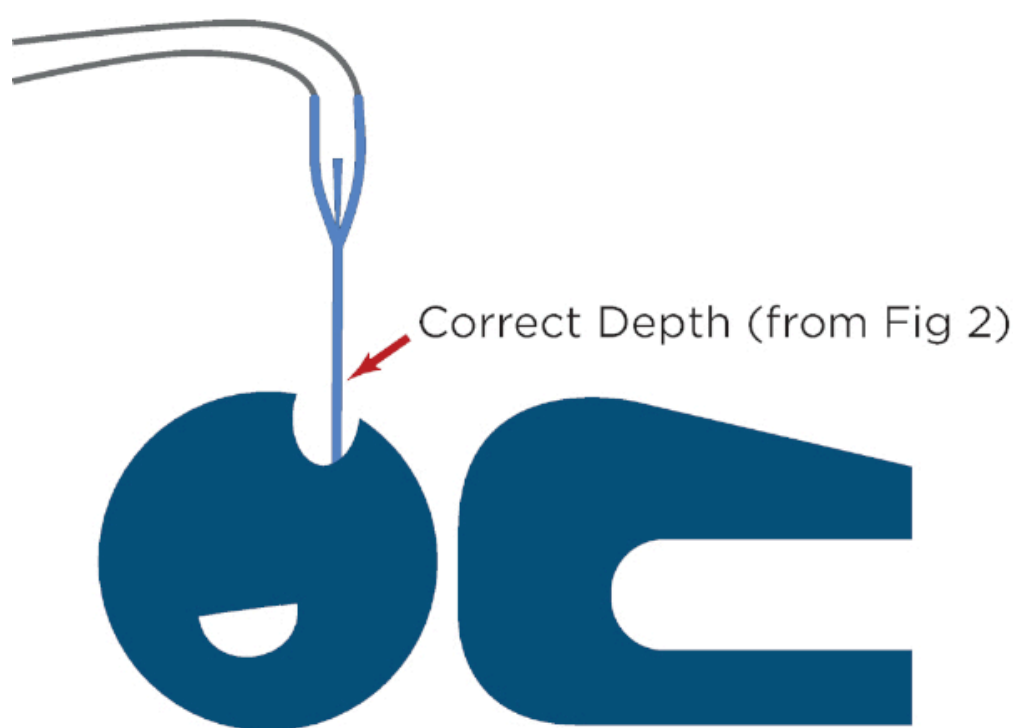

Figure 4: Advancement of the device with light pressure, until the required length of tube has been inserted. Please click here to view a larger version of this figure.

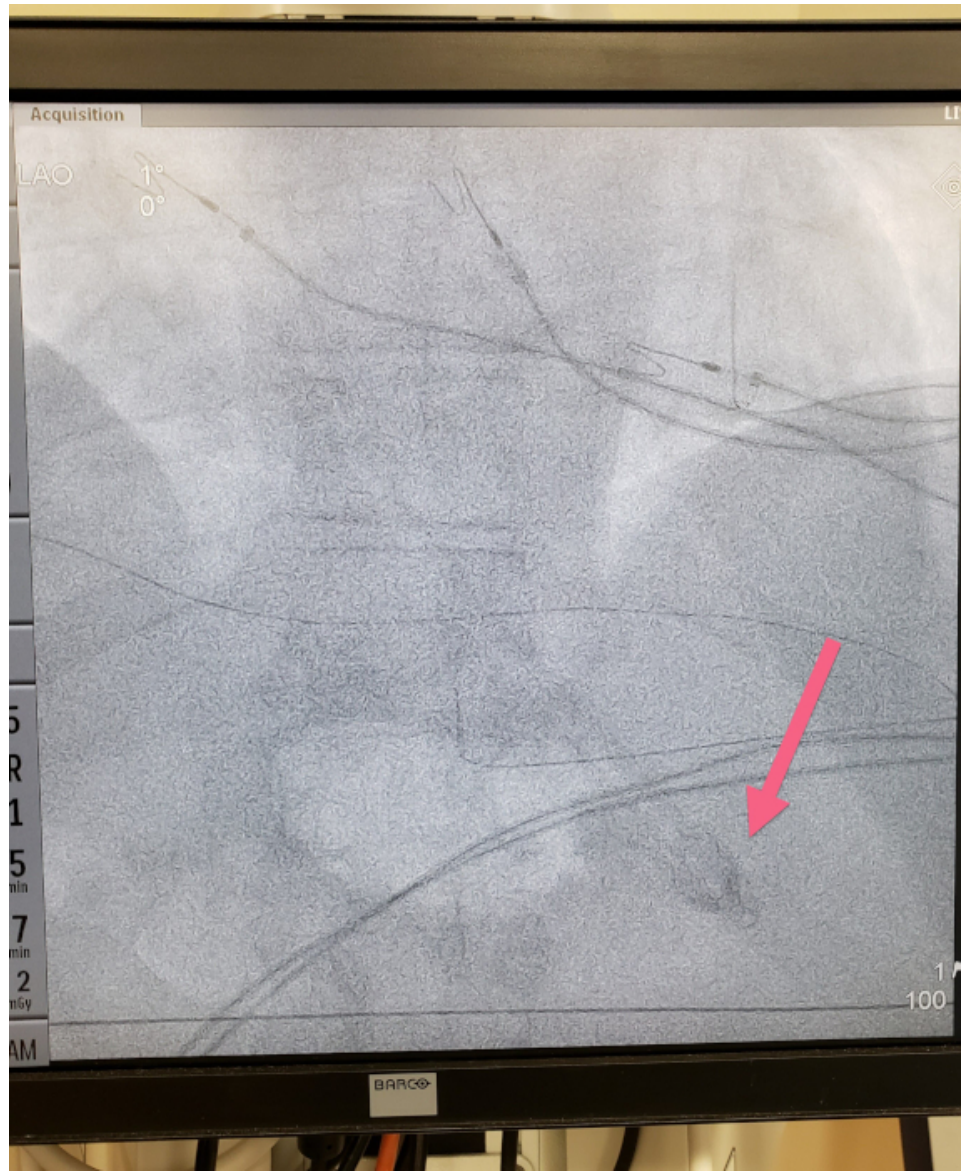

Figure 5: Fluoroscopic image demonstrating the tip of the device below the diaphragm. Please click here to view a larger version of this figure. 


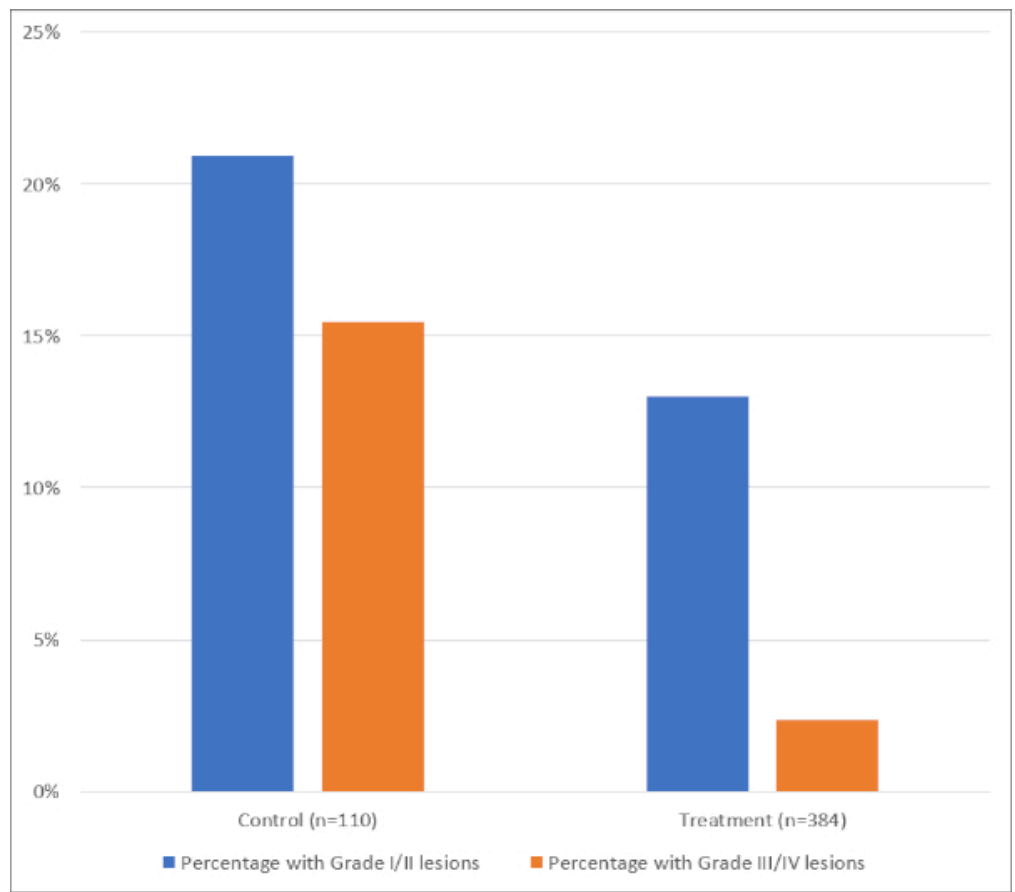

Figure 6: Summary of data from meta-analysis of studies on esophageal cooling utilizing direct liquid instillation. Please click here to view a larger version of this figure.

\begin{tabular}{|c|c|c|c|}
\hline $\begin{array}{l}\text { Endoscopy Results } \\
\qquad(\mathrm{n}=120)\end{array}$ & $\begin{array}{c}\text { Protected } \\
(n=60) \\
\end{array}$ & $\begin{array}{l}\text { Control } \\
(n=60) \\
\end{array}$ & Pvalue \\
\hline Any mucosal injury & 2 & 12 & 0.008 \\
\hline \multicolumn{4}{|l|}{ Lesion grades per group } \\
\hline No lesion & 56 & 42 & \\
\hline Grade 1 (Erythema) & 0 & 4 & \\
\hline Grade 2 (Erosion $<5$ mm) & 1 & 1 & \\
\hline Grade 3 (Erosion $(\mathrm{s})>5$ mm) & 0 & 1 & \\
\hline Grade 4a (Superficial ulcer, clean) & 1 & 4 & \\
\hline Grade 4b (Superficial ulcer with clot) & 0 & 1 & \\
\hline Grade 5a (Deep ulcer, clean) & 0 & 1 & \\
\hline Grade 5b (Deep ulcer with clot) & 0 & 0 & \\
\hline Grade 6 (Fistula) & 0 & 0 & \\
\hline Plexus Injury (Gastroparesis) & 2 & 6 & \\
\hline
\end{tabular}

Table 1: Summary of primary outcome of randomized-controlled study of dedicated esophageal cooling device.

\section{Discussion}

Modification of the placement procedure may be necessary by crimping the water outflow tube, increasing the stiffness of the heat exchange device during placement. The identification of which connecting tube is water outflow can be performed by crimping either tube and examining to see which causes the device to stiffen, and which causes the device to soften. Crimping the inlet tube will decrease water inlet flow and soften the device, crimping the outlet will increase water backpressure and stiffen it.

Limitations of this method of esophageal temperature modulation to counteract thermal injury from left atrial ablation include the inherent heattransfer limitation of any technology. Although whole-body temperature modulation can be achieved with esophageal heat exchange, there is still the potential to overcome this heat transfer capacity if sufficient energy is utilized in ablation. As such, changes from standard ablation parameters are not recommended, and usual ablation technique should be maintained. In general, the device is utilized in patients that are endotracheally intubated; however, a number of sites utilize this protocol in patients under conscious sedation without difficulty ${ }^{22}$. Finally, there remains some uncertainty as to the factors necessary for fistula formation, and aspects beyond energy exchange may be involved. 
The use of direct esophageal temperature modulation to prevent esophageal injury during atrial ablation has been used in various forms over the last several years. The most common use has been in cooling during RF ablation, using either balloon devices or direct instillation of cold fluid $^{8,9,10,11,12,13,14,15}$. More recent use has focused on warming to counteract cryothermal injury during cryoablation ${ }^{23,24,25,26}$. Use of a dedicated esophageal heat transfer device such as described in this protocol offers the advantage of targeting specific temperatures in the esophagus while avoiding the significant risks and logistical workload of direct instillation of free liquid into the GI tract.

Future applications of this method include the leverage of the known protean effects of patient temperature modulation, in particular temperature reduction ${ }^{27,28}$. Given the well-described protective effects of hypothermia on injured neurons, an additional application may involve the reduction of post-operative cognitive dysfunction ${ }^{29,30,31,32}$. Recent data in the burn literature reviewing 2,495 patients highlight the importance of cooling thermal injury in reducing burn depth, grafting, and operative requirements, noting that the mechanisms involve more than just dissipation of heat, but also the alteration of cellular behavior through decreasing release of lactate and histamine, stabilizing thromboxane and prostaglandin levels, and inhibiting kallikrein activity ${ }^{33}$. If similar mechanisms of action are involved in the esophagus, additional benefits to surrounding structures might be anticipated. Preliminary findings and anecdotal data suggest that the anti-inflammatory effects of cooling may reduce infarct size after certain subsets of myocardial injury, renal dysfunction after transplantation, the occurrence of post-operative pericarditis, and the rate of post-procedure gastroparesis ${ }^{34,35,36,37}$.

Critical steps include ensuring (a) proper placement of the heat transfer device (b) proper water temperature setpoint, and (c) continual water circulation through the heat transfer device. Proper placement of the device is readily confirmed with fluoroscopy, with particular attention towards the epigastric region near where the tip of the heat exchange device is expected to terminate. Water temperature is easily adjusted on the heat exchanger console, keeping in mind that up to 7-10 min may be needed for the circulating water to attain the setpoint temperature from the starting temperature. Continual water circulation is necessary for the device to properly transfer heat. Water circulation can be confirmed by visualization of the spinning water-flow paddle wheel present on some heat exchanger models. On heat exchanger models that lack a waterflow paddle wheel, an alarm will trigger when flow is obstructed. A potential cause of water flow obstruction is improper placement of the heat exchange device (if placed too deep, causing bending/kinking of the tube in the distal stomach, or in rarer cases, if allowed to coil up and bend in the oropharynx or proximal esophagus during placement). Troubleshooting in this case involves a simple visualization under fluoroscopy to determine placement level and adjusting as needed.

\section{Disclosures}

EK is an equity owner of Attune Medical, manufacturer of esophageal heat transfer technology. MG, PS, CT, JG, and BC serve as Principal Investigators for studies of esophageal cooling with funding to their hospital institutions, but receive no direct corporate compensation. MM has provided consulting services for Attune Medical. All other authors declare no conflicts of interest with this work.

Acknowledgments

None

\section{References}

1. Calkins, $\mathrm{H}$. et al. $2017 \mathrm{HRS/EHRA/ECAS/APHRS/SOLAECE} \mathrm{expert} \mathrm{consensus} \mathrm{statement} \mathrm{on} \mathrm{catheter} \mathrm{and} \mathrm{surgical} \mathrm{ablation} \mathrm{of} \mathrm{atrial}$ fibrillation: Executive summary. Europace. 20 (1), 157-208 (2018).

2. Han, H. C. et al. Atrioesophageal Fistula: Clinical Presentation, Procedural Characteristics, Diagnostic Investigations, and Treatment Outcomes. Circulation: Arrhythmia and Electrophysiology. 10 (11) (2017).

3. Kapur, S., Barbhaiya, C., Deneke, T., Michaud, G. F. Esophageal Injury and Atrioesophageal Fistula Caused by Ablation for Atrial Fibrillation. Circulation. 136 (13), 1247-1255 (2017).

4. Khakpour, H. et al. Atrioesophageal Fistula After Atrial Fibrillation Ablation: A single center series. Journal of Atrial Fibrillation. 10 (3), 1654 (2017).

5. Zakaria, A., Hipp, K., Battista, N., Tommolino, E., Machado, C. Fatal esophageal-pericardial fistula as a complication of radiofrequency catheter ablation. SAGE Open Medical Case Reports. 7 (2019).

6. Khan, M. Y., Siddiqui, W. J., Iyer, P. S., Dirweesh, A., Karabulut, N. Left Atrial to Esophageal Fistula: A Case Report and Literature Review. American Journal of Case Reports. 17 814-818 (2016).

7. Kadado, A. J., Akar, J. G., Hummel, J. P. Luminal esophageal temperature monitoring to reduce esophageal thermal injury during catheter ablation for atrial fibrillation: A review. Trends in Cardiovascular Medicine. 29 (5), 264-271 (2019).

8. Berjano, E. J., Hornero, F. A cooled intraesophageal balloon to prevent thermal injury during endocardial surgical radiofrequency ablation of the left atrium: a finite element study. Physics in Medicine and Biology. 50 (20), N269-279 (2005).

9. Lequerica, J. L., Berjano, E. J., Herrero, M., Hornero, F. Reliability assessment of a cooled intraesophageal balloon to prevent thermal injury during RF cardiac ablation: an agar phantom study. Journal of Cardiovascular Electrophysiology. 19 (11), 1188-1193 (2008).

10. Lequerica, J. L., Berjano, E. J., Herrero, M., Melecio, L., Hornero, F. A cooled water-irrigated intraesophageal balloon to prevent thermal injury during cardiac ablation: experimental study based on an agar phantom. Physics in Medicine and Biology. 53 (4), N25-34 (2008).

11. Arruda, M. S., Armaganijan, L., Di Biase, L., Rashidi, R., Natale, A. Feasibility and safety of using an esophageal protective system to eliminate esophageal thermal injury: implications on atrial-esophageal fistula following AF ablation. Journal of Cardiovascular Electrophysiology. 20 (11), 1272-1278 (2009).

12. Tsuchiya, T., Ashikaga, K., Nakagawa, S., Hayashida, K., Kugimiya, H. Atrial fibrillation ablation with esophageal cooling with a cooled waterirrigated intraesophageal balloon: a pilot study. Journal of Cardiovascular Electrophysiology. 18 (2), 145-150 (2007).

13. Scanavacca, M. I. et al. in European Society of Cardiology Congress. 1561 - 5 September. (2007).

14. Kuwahara, T. et al. Oesophageal cooling with ice water does not reduce the incidence of oesophageal lesions complicating catheter ablation of atrial fibrillation: randomized controlled study. Europace. 16 (6), 834-839 (2014). 
15. Sohara, H., Satake, S., Takeda, H., Yamaguchi, Y., Nagasu, N. Prevalence of esophageal ulceration after atrial fibrillation ablation with the hot balloon ablation catheter: what is the value of esophageal cooling? Journal of Cardiovascular Electrophysiology. 25 (7), 686-692 (2014).

16. John, J. et al. The effect of esophageal cooling on esophageal injury during radiofrequency catheter ablation of atrial fibrillation. Journal of Interventional Cardiac Electrophysiology. (2019).

17. Muller, P. et al. Higher incidence of esophageal lesions after ablation of atrial fibrillation related to the use of esophageal temperature probes. Heart Rhythm. 12 (7), 1464-1469 (2015).

18. Palaniswamy, C. et al. The Extent of Mechanical Esophageal Deviation to Avoid Esophageal Heating During Catheter Ablation of Atrial Fibrillation. Journal of the American College of Cardiology: Clinical Electrophysiology. 3 (10), 1146-1154 (2017).

19. Koruth, J. S. et al. Mechanical esophageal displacement during catheter ablation for atrial fibrillation. Journal of Cardiovascular Electrophysiology. 23 (2), 147-154 (2012).

20. Leung, L. W. et al. Esophageal cooling for protection during left atrial ablation: a systematic review and meta-analysis. Journal of Interventional Cardiac Electrophysiology. Nov 22 [Epub ahead of print] (2019).

21. Leung, L. W. M. et al. Improving Esophageal Protection during AF ablation: the IMPACT study. medRxiv. (2020).

22. Feher, M., Anneken, L., Gruber, M., Achenbach, S., Arnold, M. Esophageal cooling for prevention of thermal lesions during left atrial ablation procedures: a first in man case series in European Hearth Rhythm Association Congress. Lisbon, Portugal (2019).

23. Mercado-Montoya, M., MacGregor, J., Kulstad, E. Esophageal warming with an esophageal heat transfer device to limit temperature decrease during left atrial cryoablation in 12th Annual International Symposium on Catheter Ablation Techniques. Paris, France (2018).

24. Mercado-Montoya, M., Kulstad, E. Esophageal warming to prevent excessive temperature decreases during cryoablation - Abstracts from the 24th International Atrial Fibrillation Symposium Journal of Cardiovascular Electrophysiology. 30 (9), 1734-1761 (2019).

25. De Potter, T., Boersma, L., Babkin, A., Mazor, M., Cox, J. Novel Linear Cryoablation Catheter to Treat Atrial Fibrillation in Heart Rhythym Society - Scientific Sessions. Boston, MA (2018)

26. Boersma, L., Cox, J., Babkin, A., Mazor, M., De Potter, T. Treatment of Typical Atrial Flutter with a Novel Cryolinear Ablation Catheter First Experience in Heart Rhythm Society - Scientific Sessions. Boston, MA (2018).

27. Yenari, M. A., Han, H. S. Neuroprotective mechanisms of hypothermia in brain ischaemia. Nature Reviews Neuroscience. 13 (4), $267-278$ (2012).

28. Polderman, K. H. Mechanisms of action, physiological effects, and complications of hypothermia. Critical Care Medicine. 37 (7 Suppl) (2009).

29. Silveira, R. C., Procianoy, R. S. Hypothermia therapy for newborns with hypoxic ischemic encephalopathy. Jornal de Pediatria. (2015).

30. Shankaran, S. et al. Effect of depth and duration of cooling on deaths in the NICU among neonates with hypoxic ischemic encephalopathy: a randomized clinical trial. Journal of the American Medical Association. 312 (24), 2629-2639 (2014).

31. Kotekar, N., Shenkar, A., Nagaraj, R. Postoperative cognitive dysfunction - current preventive strategies. Clinical Interventions in Aging. 13 2267-2273 (2018).

32. Medi, C. et al. Subtle post-procedural cognitive dysfunction after atrial fibrillation ablation. Journal of the American College of Cardiology. 62 (6), 531-539 (2013).

33. Griffin, B. R., Frear, C. C., Babl, F., Oakley, E., Kimble, R. M. Cool Running Water First Aid Decreases Skin Grafting Requirements in Pediatric Burns: A Cohort Study of Two Thousand Four Hundred Ninety-five Children. Annals of Emergency Medicine. (2019).

34. Niemann, C. U. et al. Therapeutic Hypothermia in Deceased Organ Donors and Kidney-Graft Function. New England Journal of Medicine. 373 (5), 405-414 (2015).

35. Erlinge, D. et al. Therapeutic hypothermia for the treatment of acute myocardial infarction-combined analysis of the RAPID MI-ICE and the CHILL-MI trials. Therapeutic Hypothermia and Temperature Management. 5 (2), 77-84 (2015).

36. Matsui, T., Yoshida, Y., Yanagihara, M., Suenaga, H. Hypothermia at 35 degrees C Reduces the Time-Dependent Microglial Production of Pro-inflammatory and Anti-inflammatory Factors that Mediate Neuronal Cell Death. Neurocritical Care. (2013).

37. Horiguchi, A. et al. Abstract 11134: Esophagus Temperature Monitoring Predicts Gastric Hypoperistalsis After Catheter Ablation for Atrial Fibrillation. Circulation. 140 (Suppl_1), A11134-A11134 (2019). 